\title{
Ibuprofen, paracetamol, and steam for patients with respiratory tract infections in primary care: pragmatic randomised factorial trial
}

\begin{abstract}
Objective To assess strategies for advice on analgesia and steam inhalation for respiratory tract infections.

Design Open pragmatic parallel group factorial randomised controlled trial.

Setting Primary care in United Kingdom.

Participants Patients aged $\geq 3$ with acute respiratory tract infections.

Intervention 889 patients were randomised with computer generated random numbers in pre-prepared sealed numbered envelopes to components of advice or comparator advice: advice on analgesia (take paracetamol, ibuprofen, or both), dosing of analgesia (take as required $v$ regularly), and steam inhalation (no inhalation $v$ steam inhalation).

Outcomes Primary: mean symptom severity on days 2-4; symptoms rated 0 (no problem) to 7 (as bad as it can be). Secondary: temperature, antibiotic use, reconsultations.

Results Neither advice on dosing nor on steam inhalation was significantly associated with changes in outcomes. Compared with paracetamol, symptom severity was little different with ibuprofen (adjusted difference $0.04,95 \%$ confidence interval -0.11 to 0.19 ) or the combination of ibuprofen and paracetamol $(0.11,-0.04$ to 0.26$)$. There was no evidence for selective benefit with ibuprofen among most subgroups defined before analysis (presence of otalgia; previous duration of symptoms; temperature $>37.5^{\circ} \mathrm{C}$; severe symptoms), but there was evidence of reduced symptoms severity benefit in the subgroup with chest infections (ibuprofen $-0.40,-0.78$ to -0.01 ; combination -0.47 ; -0.84 to -0.10 ), equivalent to almost one in two symptoms rated as a slight rather than a moderately bad problem. Children might also benefit from treatment with ibuprofen (ibuprofen: $-0.47,-0.76$ to -0.18 ; combination: $-0.04,-0.31$ to 0.23 ). Reconsultations with new/unresolved
\end{abstract}

symptoms or complications were documented in $12 \%$ of those advised to take paracetamol, $20 \%$ of those advised to take ibuprofen (adjusted risk ratio $1.67,1.12$ to 2.38 ), and $17 \%$ of those advised to take the combination (1.49, 0.98 to 2.18$)$. Mild thermal injury with steam was documented for four patients $(2 \%)$ who returned full diaries, but no reconsultations with scalding were documented.

Conclusion Overall advice to use steam inhalation, or ibuprofen rather than paracetamol, does not help control symptoms in patients with acute respiratory tract infections and must be balanced against the possible progression of symptoms during the next month for a minority of patients. Advice to use ibuprofen might help short term control of symptoms in those with chest infections and in children.

Trial registration ISRCTN 38551726.

\section{Introduction}

Acute respiratory tract infections are the commonest acute condition managed in primary care, and control of symptoms is central for patients and parents of affected children. ${ }^{12}$ Advice to use antipyretics or analgesics is perhaps the commonest advice given in the National Health Service (NHS) ${ }^{3}$ but use is often suboptimal even for conditions with both fever and pain (such as otitis media). ${ }^{4}$

A previous trial in primary care showed that ibuprofen is more effective than paracetamol alone for resolution of fever in children, ${ }^{56}$ and this was supported by more recent systematic reviews. ${ }^{78}$ The effect sizes, however, were modest (particularly for children); most trial data provide short term assessment, and limited assessment of symptom control or progression; and the study designs are normally tightly controlled efficacy designs, often in secondary care - all of which makes it difficult to apply 
findings in primary care, where most advice on analgesia is given. There are also concerns about inhibiting the inflammatory/febrile process ${ }^{9}$ and about the adverse effects of ibuprofen on cardiovascular risk in adults..$^{10}$ Alternate dosing of paracetamol and ibuprofen is common and was more effective in children in one trial, ${ }^{11}$ but the trial was a less pragmatic efficacy design, and there is little evidence in adults. A recent systematic review concluded that there might be marginal benefit in combining analgesics but also noted short term effects of marginal clinical importance and secondary care settings. ${ }^{12}$ The National Institute for Health and Clinical Excellence (NICE) cautioned against combined use because of renal/hepatic complications, ${ }^{3}$ although the evidence was largely from case studies in a secondary care setting.

Although advice to use steam is commonly given in primary care, the evidence of effectiveness is limited ${ }^{13}$ as trials have been small. There have been some reports of burns, ${ }^{14}$ which are rare and have occurred in children using boiling water after poor instruction, so the NHS direct website recommended that doctors advise parents to "sit in the bathroom with a hot shower running."

We estimated the effectiveness of commonly used strategies for advice in analgesia and steam inhalation.

\section{Method}

\section{Design and randomisation}

We carried out a pragmatic randomised factorial trial in a primary care setting in patients with respiratory tract infection. We examined the difference in the effectiveness between three different antipyretic regimens: ibuprofen, paracetamol, and combined ibuprofen and paracetamol; whether regular antipyretic dosing gives significantly better control of symptoms and temperature than "as required" dosing; and whether regular inhalation with steam improves symptom control. Further details are in the appendix.

A statistician independent of the study team coordinated the randomisation, which was stratified by antibiotic prescribing strategy (immediate antibiotics or delayed antibiotics). Patients were randomised to one of 12 advice groups defined by three factors (three drug groups $\times$ two dosing groups $\times$ two steam groups) in a factorial design, with computer generated random number tables to determine one of 12 advice slips contained in sealed numbered envelopes. The advice factors were:

- Drugs: advice to use paracetamol or ibuprofen or combined paracetamol and ibuprofen alternating

- Dosing advice to use drugs regularly four times a day for at least three days then ad lib or to use on an as required basis, both as per guidance in the British National Formulary (BNF, 59th edition). The doses used each day were recorded in a diary

- Steam-advice to inhale with steam for at least 15 minutes (five minutes three times a day) or asked not to use steam. The number of times steam inhalation was done was recorded in a daily diary.

A key logistic issue in complex pragmatic factorial trials is the avoidance of errors in delivery of the intervention. We made it simple to execute randomisation as the clinician took the next pack off the shelf that contained pre-randomised advice sheets (that is, there was no requirement (hence minimal error) in finding and using the correct advice sheets). With careful attention to practitioner equipoise this method of randomisation to different advice strategies has proved successful previously, ${ }^{415}$ and there was no evidence of subversion either from the order of envelope use or patients' characteristics. General practitioners were asked to complete non-recruitment logs and also a questionnaire at the end of the study documenting reasons for non-recruitment of eligible patients and for eligible patients who declined.

\section{Inclusion criteria}

We included patients aged $\geq 3$ presenting to a general practitioner or nurse in primary care with a respiratory tract infections diagnosed by the health professional (acute cold; influenza; sore throat; otitis media; sinusitis; croup; cough/lower respiratory tract infection). There was no clinical selection other than the inclusion/exclusion criteria: practitioners were asked to include consecutive patients whenever feasible.

\section{Exclusion criteria}

We excluded patients with asthma (unless it was not sensitive to ibuprofen or aspirin); peptic ulcer; hypersensitivity to analgesics; inability to complete outcomes (such as visually impairment, psychosis, severe depression); conditions requiring hospital admission (such as meningitis, severe pneumonia, epiglottitis, Kawasaki disease, etc); or immunodeficiency; and pregnant or breastfeeding women.

\section{Outcomes}

\section{Primary outcome}

Our primary outcome was symptom control documented as mean symptom severity measured at the end of each day, averaged over days two to four of a two week symptom diary (the first few days are when symptoms of all respiratory tract infections are at their worst $\left.{ }^{4}{ }^{16}\right)$. The diary was completed by patients (and/or children, with their parents helping as necessary) until symptoms returned to normal, using previously validated formats ${ }^{16}{ }^{17}$ for rating symptoms $(0=$ no problem, $6=$ as bad as it could be for feeling generally unwell, sleep disturbance, fever, interference with normal activities, sore throat, cough, short of breath, facial/sinus pain, earache, and runny/blocked nose). Though we have reported symptom resolution (duration of symptoms rated moderately bad or worse from the diary or brief questionnaire ${ }^{18}$ ), this was not specified initially as an outcome as duration differs according to the particular respiratory infection. ${ }^{19}$

Patients were telephoned (on day two to three) to check for any problems with diary completion. If we had not received a diary after two weeks, we sent one mailed reminder and then phoned as necessary to document outcomes using a brief questionnaire, which we have shown to be reliable. ${ }^{15}$

\section{Secondary outcome measures}

Secondary outcomes included:

- Side effects (rash, diarrhoea, vomiting, abdominal pain) documented in the diary; scalding was not included in the initial protocol but was included in subsequent protocol revisions

- Mean morning and evening temperature reading with Tempadot (3M, Bracknell) thermometers (orally where possible)

- Antibiotic use documented in the diary (which was not initially specified as an outcome for this component of the trial)

- Return visit with new or worsening symptoms or complications of intervention, ${ }^{18}$ defined as a return with a 
symptom or diagnosis of respiratory tract infection recorded with a structured proforma by a member of the research team. "Complications" were defined as a new consultation documented in the notes within 28 days with otitis media, sinusitis, pneumonia, quinsy, cervical adenitis, meningitis, or septicaemia. This outcome was not specified as an effectiveness outcome in the protocol but is routinely collected to document harms.

\section{Sample size calculation}

We calculated our required sample size for $\alpha=0.05$ and $\beta=0.2$ using the NQuery multiple group sample size programme. We assumed an SD of 1.1 for our primary outcome. ${ }^{15-17}$ Our assumption was that each factor was a separate "experiment" - that is, that factors were independent and that the comparisons of interest were within each factor. For the analgesic factor we assumed that either ibuprofen or the combined group could have symptom control $0.5 \mathrm{SD}$ better than the paracetamol only group. We need 228 patients to allow for 12 groups and $80 \%$ follow-up, or 504 patients for 0.33 SD (equivalent to one in three rating symptoms mild versus moderate, agreed by previous consensus as a modest effect ${ }^{16}$ ). The limiting element of the sample size calculation, however, was for the antibiotic component of the study (reported elsewhere): we estimated 72 patients per group or 450 altogether in the no and delayed prescribing groups, allowing for $20 \%$ loss to follow-up and assuming $15 \%$ use of antibiotics in the no antibiotic group, and that antibiotic use ranged between $20 \%$ and $35 \%$ in the delayed groups $(20 \%, 25 \%, 35 \%, 35 \%)$. The trial was not specifically powered to detect interactions between the intervention factors nor for subgroups.

\section{Analysis}

We used analysis of covariance for a factorial study for the main continuous outcomes, baseline symptom severity, and potential confounders as appropriate (in this case smoking was included in the models as it is related to symptom severity). All results presented also control for antibiotic prescribing strategy, although the estimates were little altered. Cox regression was used for the duration of symptoms (after we checked the proportional hazards assumption) and logistic regression for antibiotic use and reconsultation. Odds ratios were converted to risk ratios with standard formulas. ${ }^{20} \mathrm{We}$ present the "main effects" of each factor with $95 \%$ confidence intervals as we did not find significant interactions. The primary analysis was intention to treat with complete data (that is, no matter whether patients complied, their data were analysed according to randomisation group) with no imputation of missing data. The trial management group defined important subgroups of interest in advance of the analysis (but not at study inception): a per protocol analysis for analgesics (that is, patients taking the correct analgesics); patients with lower respiratory tract infection (with a longer course of more severe illness) ${ }^{16}$; patients with otitis media (a more painful illness) ${ }^{4}$; baseline severity of symptoms above the median; baseline temperature $37.5^{\circ} \mathrm{C}$ or above $^{21}$; previous duration longer than the median; and children (age $\leq 16$ ). For temperature control we also specified the subgroup of patients who reported fever in the previous 24 hours. As part of responding to referees we also checked for interactions according to older age (age $\geq 60$ ) and previous lung problems. For the clinical subgroups evidence of effectiveness in subgroups was agreed as requiring both a significant interaction term and significant estimates when the subgroup was selected. No interim analyses were performed.

\section{Results}

From 3 March 2010 to 28 March 2012, 53 general practitioners and practice nurses recruited 889 patients in 25 practices (figure) $\Downarrow$. Baseline characteristics were similar in all groups (table $1 \Downarrow$ ) and similar for those for whom follow-up data were available (see table B in appendix). All patients were followed up for one month; further follow-up after the initial month was 0.84 years (SD 0.36) and was similar in all groups. The diary symptom severity scale had acceptable internal reliability for days two to four (Cronbach's $\alpha 0.72$ for day two, 0.79 for day three, and 0.79 for day four) and was sensitive to change over the week (standardised response mean 1.60). Non-recruitment logs were poorly completed by general practitioners.

The non-recruitment questionnaire had responses from 20 respondents who recruited most $(n=704)$ of the patients. The top reason (given by 14 recruiters) for eligible patients declining was that the patient was too busy or insufficiently interested, followed by concern about the proposed drug, then by the patient being too unwell. The top reason given for not approaching eligible patients (given by 18/19 recruiters) was insufficient time.

\section{Attrition bias}

The characteristics of patients in whom the primary outcome was not documented were similar to those followed up $(n=751)$ for baseline symptom score ( $1.0 v 0.98$, respectively), female $\operatorname{sex}(82 / 136(60 \%) v 458 / 744(62 \%))$, age $\leq 16(31 / 136(23 \%)$ $v 200 / 744(27 \%)$ ), diagnosis of lower respiratory tract infection $(22 / 135(16 \%) v 113 / 742(15 \%))$ or diagnosis otitis media $(16 / 134(12 \%) v 66 / 740(9 \%))$.

\section{Performance bias: compliance during first three days}

From the main diary data for paracetamol use, patients took a mean of 4.2 doses a day for the first three days in the paracetamol group, 3.5 in the combined group, and 0.4 in the ibuprofen group. For ibuprofen, patients took a mean of 0.3 doses in the paracetamol group, 2.9 in the ibuprofen group, and 2.7 in the combined group. Steam inhalation was reported a mean of 1.6 times a day in the steam group for days two and three and 0.1 times in the no steam group. Advice to use analgesic regularly compared with as required made little difference to the amount of analgesia used for either paracetamol (2.8 doses $v 2.4$ doses) or ibuprofen ( 2.0 doses $v 1.9$ doses).

\section{Symptom control: main results}

There were no interactions between interventions (see appendix for details). Overall, compared with advice to use paracetamol alone, alternative advice on analgesia made little difference to symptom severity (ibuprofen $0.04,95 \%$ confidence intervals -0.11 to 0.19 ; combined $0.11,-0.04$ to 0.26 ) (table $2 \Downarrow$ ). There was also no effect of steam (table $3 \Downarrow$ ) and no effect of advice on regular compared with as required dosing (see appendix table A), and the per protocol analysis produced similar results.

\section{Primary outcome: subgroups}

There were no significant interactions for subgroups defined by the trial team except for lower respiratory tract infection and for children (see appendix for details) nor for subgroups suggested during the review process (age $\geq 60$, previous lung problems). When we selected the lower respiratory tract infection subgroup, the estimate of benefit was a reduction of 0.40 (95\% confidence interval -0.78 to -0.01$)$ in symptom 
severity for the ibuprofen group and $0.47(-0.84$ to -0.10$)$ for the combined group (table $2 \Downarrow$ ), roughly equivalent to one person in two rating their symptoms a slight rather than a moderately bad problem. When we selected the subgroup of patients aged $\leq 16$, the benefit from ibuprofen in symptom severity again a reduction of 0.47 ( -0.76 to -0.18$)$. Those aged over 16 might even have less good symptom control (table 2), although the effect was small.

\section{Harms}

The main harm anticipated from analgesic use was with ibuprofen: abdominal side effects or wheeze in individuals with undetected asthma. The main diary was the only source of these data, but there was little evidence of these anticipated harms from ibuprofen, with similar figures in all groups (table 4) $\downarrow$ ).

There were no other harms reported for the drug components, either by participants or based on notes review. The main harm anticipated from steam inhalation was mild scalding: this was reported by four of the 207 (2\%) patients in the steam group who provided usable symptom data from the full diary, but this was not severe enough to require a consultation. Tables 5 and 6 show effectiveness of advice on analgesia and steam on other outcomes. $\Downarrow \Downarrow$ Return with new or unresolved symptoms or complications occurred in $12 \%$ of the paracetamol group but were more common with ibuprofen $(20 \%)$ or the combination (17\%) table $5 \Downarrow$ ). Most of the 17 “complications" recorded were not serious, and three could be classified as reconsultations

based on the baseline case record form: two in the paracetamol group (one cellulitis, one otitis media); 11 in the ibuprofen group (one quinsy, three sinusitis, one meningitis, one pneumonia, three new cases of otitis media, and two reconsultations with otitis media); and four in the combined group (one quinsy, one new case of sinusitis, one reconsultation with sinusitis, one cervical adenitis).

\section{Discussion \\ Principal findings}

In this pragmatic randomised factorial trial in patients with respiratory tract infections in primary care, patients gained no benefit from advice to use ibuprofen alone or a combination of ibuprofen and paracetamol nor advice to use regular (rather than as required) analgesia or steam inhalation. Patients with chest infections and/or children probably did get more symptomatic relief from ibuprofen alone, but this must be balanced against possible modest harms - an increase in return visits with new symptoms, unresolved symptoms, or a complication, although most complications were not serious.

\section{Strengths and weaknesses}

To our knowledge this is the largest trial to date assessing the impact of analgesic/antipyretic use and steam inhalation on control and progression of symptoms. The patient groups were broadly defined with a range of respiratory infections so the increased variance in outcome measures makes type II error more likely. The primary outcome (symptom severity in the first few days), however, should be applicable in all acute infections and was reasonably reliable and sensitive to change in these patients. Recruitment was slow considering the incidence of respiratory infections, but feedback during and after the study made it clear that this study was easy to recruit to, and the limit to recruitment was time pressures in busy winter clinics, as in previous studies, ${ }^{15}{ }^{16}$ confirmed by the end of study questionnaire, hence non-recruitment logs were poorly completed. This raises questions of generalisability, though there was no evidence that the patients with more severe symptoms were excluded as comparison with recent large simple observational studies and trials shows that, if anything, our included patients were slightly more severely affected (on a 0-3 scale, the mean severity for sore throat at baseline was 2.4 in the current study versus 2.0 in the DESCARTE study; for cough was 2.6 in the current study versus 2.2 in GRACE studies ${ }^{18} 22$ ). Type I error is a possibility, particularly for the subgroup analyses that were not specified at study inception, but subgroups were defined before we performed the analysis, and there were highly significant interactions for two significant subgroups. Although the factorial design allowed us to explore possible interactions between interventions, we did not power this study specifically to detect interactions between the factors; the sample sizes should have been sufficient to detect interactions of the order of 0.5 of a standardised effect size (that is, moderate and equivalent to one out of two symptoms being rated a slight rather than a moderate effect). The results controlled for potential confounders, but as estimates were not meaningfully altered, the impact of confounding is likely to be limited. This was a pragmatic study so patients were free not to comply with advice, but in fact compliance was reasonably good, except that there was little impact of advising patients to take analgesics regularly. The consent process (where patients were asked to use the strategy they would be randomised to) and the information sheets provided are likely to provide higher compliance than would occur in everyday practice. The grouping of diagnostic categories (such as chest infections) was based on previous definitions of lower respiratory tract infections ${ }^{18} 23$ applied to general practitioners' recording of the diagnosis or problem in the case record form, but diagnostic labels are likely to be variable.

\section{Results in context of other studies}

There was no effect of either dosing advice or advice to inhale with steam on symptom severity-neither the estimates nor the lower confidence limits reached the defined level for clinical significance-and no intervention modified antibiotic use. Patients with uncomplicated lower respiratory infections were more likely to benefit from advice to use ibuprofen, and a recent trial showed that ibuprofen was as effective or possibly more effective than antibiotics (Carl Llor, personal communication). Children might also selectively benefit from ibuprofen. Those aged 17 and over had slightly worse symptom control with ibuprofen, although the effect was small and did not reach the threshold for clinical significance. We could not show superiority of the combined strategy, unlike a previous trial in children, ${ }^{11}$ but our trial had a pragmatic open design and was not an efficacy study, which is more likely to provide realistic estimates of effectiveness in daily practice. We could not confirm any benefit for temperature control either overall or in subgroups of patients with a higher temperature, unlike a previous efficacy trial in children. ${ }^{5}$ This could be because our trial population was much less selected and we used a broader measure (mean temperatures for three days) rather than time to fever resolution.

The main harm with ibuprofen seemed not to be gastrointestinal side effects but an increased likelihood of reconsultation with new or non-resolving symptoms. Most complications were not serious and were mainly self limiting, but there were three more serious complications in the ibuprofen group (pneumonia, meningitis, and quinsy). Although there is concern that interfering with fever can inhibit the immune response, a Cochrane review found insufficient evidence of adverse events 
to confirm or refute the concern. ${ }^{9}$ Previous observational studies ${ }^{24} 25$ have shown an association between ibuprofen use and the development of necrotising fasciitis in children with chicken pox, which could possibly be mediated by impaired neutrophil function induced by non-steroidal anti-inflammatory drugs. The ibuprofen use in the latter studies, however, could equally have been a proxy marker for more severe illness. Although the finding of increased reconsultation could have been caused by chance, there was a similar effect of borderline significance in the combined group and adjustment for possible confounders did not modify the effect, so the possibility of modest harm with ibuprofen should probably not be dismissed.

There was no evidence that steam inhalation provided symptomatic benefit, and this study was much larger than the combined studies in the Cochrane review, which showed modest benefit. ${ }^{13}$ We also recorded a few cases in which mild thermal injury was reported, which supports previous concerns about scalding, ${ }^{14}$ although there was no evidence of scalding severe enough to require a consultation.

\section{Implications for clinicians}

Clinicians should probably not advise patients to use steam inhalation in daily practice as it does not provide symptomatic benefit for acute respiratory infections and a few individuals are likely to experience mild thermal injury. Similarly, routinely advising regular rather than as required analgesia is also not likely to be effective. For most patients, clinicians should not offer ibuprofen rather than paracetamol as there is little benefit, but they could consider ibuprofen use in patients with chest infections and those aged $<16$ who might selectively benefit. Clinicians and patients need to balance any benefit from using ibuprofen against the possible harm of increasing reconsultations with poorly controlled symptoms and a small risk of complications.

We are grateful to all the general practitioners and practice nurses and particularly the patients who gave their time to this study.

PIPS Investigators

University of Southampton: Paul Little, Ian Williamson, Mike Moore, Mark Mullee, Jo Kelly, Julie Hooper, Lisa McDermott, Gerry Leydon, Katherine Salisbury*, Emily Edwards* ${ }^{*}$, Jennifer White* ${ }^{*}$ Adam Nicholls*, Hannah Ebdon ${ }^{*}$, Susannah Gant* $\left({ }^{*}\right.$ medical students at the time); Samantha Hall, Patient and Public (PPI) representative.

Contributors: All authors and Daniel Lane contributed significantly to the development of the protocol. All authors contributed to overseeing the management of the study, agreeing the analysis plan, and to the write-up of the paper. KS, EE, JW, AN, HE, and SG developed and piloted the initial study documents supervised by the trial team; PL had the initial idea, led the grant application, and the initial drafting of the paper and is guarantor. PL, MM, and BS performed the analysis. JK and $\mathrm{JH}$ performed day to day management of the study supervised by PL. Alastair Hay provide helpful comments on the analysis approach and the manuscript. All authors had full access to all of the data (including statistical reports and tables) in the study and can take responsibility for the integrity of the data and the accuracy of the data analysis. Samantha Hall, PPI representative, kindly commented on the protocol and outcomes, and contributed to steering meetings

Funding: This study was funded by the National Institute for Health Research Programme Grants for Applied Research programme. This article presents independent research funded by the National Institute for Health Research (NIHR) under its Programme Grants for Applied Research programme (grant ref No RP-PG-0407-10098). The views expressed are those of the author(s) and not necessarily those of the NHS, the NIHR or the Department of Health.
Competing interests: All authors have completed the ICMJE uniform disclosure form at www.icmje.org/coi_disclosure.pdf and declare: no support from any organisation for the submitted work; no financial relationships with any organisations that might have an interest in the submitted work in the previous three years; no other relationships or activities that could appear to have influenced the submitted work.

Ethical approval: The study was approved by the Southampton and Southwest MREC number 06/Q1702/154 and all participants gave informed consent.

Declaration of transparency: PL affirms that the manuscript is an honest, accurate, and transparent account of the study being reported; that no important aspects of the study have been omitted; and that any discrepancies from the study as planned (and, if relevant, registered) have been explained.

Data sharing: No additional data available.

1 Kai J. Parents information needs and difficulties in coping with illness in pre-school children: a qualitative study. BMJ 1996;313:987-90.

2 Cornford CS. Why patients consult when they cough: a comparison of consulting and non-consulting patients. Br J Gen Pract 1998;48:1751-4.

3 Feverish illness in children. Assessment and initial management in children younger than 5 years. NICE Clinical Guideline 160, 2013.

4 Little P, Gould C, Williamson I, Moore M, Warner G, Dunleavey J. A pragmatic randomised controlled trial of two prescribing strategies for acute otitis media. BMJ 2001;322:336-42.

5 Hay A, Costelloe C, Redmond N, Montgomery A, Fletcher M, Hollinghurst S, et al. Paracetamol plus ibuprofen for the treatment of fever in children (PITCH): randomised controlled trial. BMJ 2008; doi:10.1136/bmj.a1302

6 Hay A, Redmond N, Fletcher M. Antipyretic drugs for children. BMJ 2006;doi:10.1136/ bmj.333.7557.4

7 Pierce C, Voss B. Efficacy and safety of ibuprofen and acetaminophen in children and adults: a meta-analysis and qualitative review. Ann Pharmacother 2010;44:489-506.

8 Southeya E, Soares-Weiser K, Kleijnenc J. Systematic review and meta-analysis of the clinical safety and tolerability ofibuprofen compared with paracetamol in paediatric pain and fever. Current Med Res Opinion 2009;25:2207-22.

9 Meremikwu M, Oyo-Ita A. Paracetamol versus placebo or physical methods for treating fever in children. Cochrane Database Syst Rev 2009;2:CD003676.

10 Trelle S, Reichenbach S, Wandel S, Hildebrand P, Tschannen B, Villiger P, et at. Cardiovascular safety of non-steroidal anti-inflammatory drugs: network meta-analysis. BMJ 2011;342:c7086

11 Sarrell E, Wielunsky E, Cohen H. Antipyretic treatment in young children with fever: acetaminophen, ibuprofen, or both alternating in a randomized, double-blind study. Arch Pediatr Adolesc Med 2006;160:197-202.

12 Pursell E. Systematic review of studies comparing combined treatment with paracetamol and ibuprofen, with either drug alone. Arch Dis Child 2011;96;1175-9.

13 Singh M. Heated, humidified air for the common cold. Cochrane Library 2004;2:CD001728.

14 Murphy S, Murray D, Smith S, Orr D. Burns caused by steam inhalation for respiratory tract infections in children. BMJ 2004;328:757.

15 Little PS, Williamson I, Warner G, Gould C, Gantley M, Kinmonth AL. An open randomised trial of prescribing strategies for sore throat. BMJ 1997;314:722-7.

16 Little P, Rumsby K, Kelly J, Watson L, Moore M, Warner G, et al. Information leaflet and antibiotic prescribing strategies for acute lower respiratory tract infection: a randomised controlled trial. JAMA 2005;293:3029-35

17 Watson L, Little P, Williamson I, Moore M, Warner G. Validation study of a diary for use in acute lower respiratory tract infection. Fam Pract 2001;18:553-4.

18 Little P, Stuart B, Moore M, Coenen S, Butler C, Godycki-Cwirko M, et al. Amoxicillin for acute lower-respiratory-tract infection when pneumonia is not suspected clinically: a 12-country, randomised, placebo-controlled trial. Lancet Infect Dis 2013;13:1323-9.

19 NICE guideline development group. Prescribing of antibiotics for self-limiting respiratory tract infections in adults and children in primary care. http://guidance.nice.org.uk/CG69/ Guidance/pdf/English

20 Zhang J, Yu K. What's the relative risk? A method of correcting the odds ratio in cohort studies of common outcomes. JAMA 1998:280:1690-1.

21 Little P, Gould C, Moore M, Dunleavey J. Predictors of poor outcome and benefits from antibiotics in children with acute otitis media: pragmatic randomised trial. BMJ 2002;325:22.

22 Butler C, Hood K, Verheij T, Little P, Melbye H, Nuttall J, et al. Variation in antibiotic prescribing and its impact on recovery in patients with acute cough in primary care: prospective study in 13 countries. BMJ 2009;b2242.

23 MacFarlane J, Holmes W, Gard P, MacFarlane R, Rose D, Weston V, et al. Prospective study of the incidence, aetiology and outcome of adult lower respiratory tract illness in the community. Thorax 2001;56:109-14.

24 Zerr DM, Alexander ER, Duchin JS, Koutsky LA, Rubens CE. A case-control study of necrotizing fasciitis during primary varicella. Pediatrics 1999;103:783-90.

25 Lesko SM, O'Brien KL, Schwartz B, Vezina R, Mitchell AA. Invasive group A streptococcal infection and nonsteroidal antiinflammatory drug use among children with primary varicella. Pediatrics 2001;107:1108-15.

Accepted: 23 September 2013

\section{Cite this as: BMJ 2013;347:f6041}

This is an Open Access article distributed in accordance with the Creative Commons Attribution Non Commercial (CC BY-NC 3.0) license, which permits others to distribute, remix, adapt, build upon this work non-commercially, and license their derivative works on different terms, provided the original work is properly cited and the use is non-commercial. See: http://creativecommons.org/licenses/by-nc/3.0/. 


\section{What is already known on this topic}

Ibuprofen is probably more effective for fever resolution in children than paracetamol alone, and the combination of both is also probably marginally better than either alone

The effects are modest, however, and it is not clear whether overall symptom control is improved and whether the existing evidence applies in typical primary care settings where patients probably have milder infections than those in secondary care

The evidence for steam inhalation is limited both for benefits and for harms

\section{What this study adds}

Advice to use steam inhalation or ibuprofen rather than paracetamol or advice to use regular rather than as required dosing are unlikely to help most patients with acute respiratory tract infections

Advice to use ibuprofen might help short term symptom control for patients with chest infections and children but must be balanced against the possible progression of symptoms during the next month in a minority of patients 


\section{Tables}

Table 1| Baseline characteristic of groups of patients with acute respiratory tract infections according to randomised treatment advice. Figures are numbers* (percentage) of patients unless otherwise specified

\begin{tabular}{lccccccc} 
& Paracetamol & Ibuprofen & Both & Drug taken as required & Drug taken regularly & No steam & Steam \\
$\begin{array}{l}\text { Mean (SD) previous duration of illness } \\
\text { (days) }\end{array}$ & $8.0(7.9)$ & $7.3(6.7)$ & $7.2(6.5)$ & $7.4(6.2)$ & $7.6(7.9)$ & $7.4(7.1)$ & $7.6(7.0)$ \\
\hline Mean (SD) severity (all symptoms) & $1.0(0.44)$ & $1.0(0.46)$ & $1.0(0.44)$ & $1.0(0.44)$ & $1.0(0.46)$ & $1.0(0.45)$ & $1.0(0.44)$ \\
\hline Fever in previous 24 hours & $180 / 304(59)$ & $182 / 291(62)$ & $166 / 285(58)$ & $268 / 443(61)$ & $260 / 437(60)$ & $287 / 453(63)$ & $241 / 427(56)$ \\
\hline Lower respiratory infection (LRTI) $\dagger$ & $38 / 263(14)$ & $47 / 260(18)$ & $50 / 254(20)$ & $70 / 388(18)$ & $65 / 389(17)$ & $65 / 401(16)$ & $70 / 376(19)$ \\
\hline Otitis media† & $27 / 302(9)$ & $26 / 290(9)$ & $29 / 283(10)$ & $43 / 441(10)$ & $39 / 434(9)$ & $40 / 453(9)$ & $42 / 422(10)$ \\
\hline Previous lung problems & $56 / 300(19)$ & $48 / 293(16)$ & $40 / 284(14)$ & $79 / 443(18)$ & $65 / 434(15)$ & $69 / 452(15)$ & $75 / 425(18)$ \\
\hline Sex (female) & $195 / 304(64)$ & $185 / 291(64)$ & $160 / 285(56)$ & $269 / 444(61)$ & $271 / 437(62)$ & $290 / 453(64)$ & $250 / 427(59)$ \\
\hline Current smoker & $48 / 304(16)$ & $57 / 290(20)$ & $55 / 285(19)$ & $72 / 442(16)$ & $88 / 437(20)$ & $83 / 452(18)$ & $77 / 427(18)$ \\
\hline Mean (SD) age (years) & $34(21)$ & $34(21)$ & $33(22)$ & $34(21)$ & $33(22)$ & $35(21)$ & $32(22)$ \\
\hline
\end{tabular}

*Denominators vary because of missing data.

†LRTI and otitis media based on diagnosis/symptoms recorded by general practitioner in CRF=case report form. 
Table 2| Effectiveness of advice strategies for analgesia on symptom control in patients with acute respiratory tract infections. Figures are crude mean symptom severity scores two to four days after seeing doctor

\begin{tabular}{|c|c|c|c|c|c|}
\hline & \multirow[b]{2}{*}{$\begin{array}{c}\text { Paracetamol } \\
\text { crude mean (SD) }\end{array}$} & \multicolumn{2}{|c|}{ Ibuprofen } & \multicolumn{2}{|c|}{ Paracetamol and ibuprofen } \\
\hline & & Crude mean (SD) & $\begin{array}{l}\text { Adjusted }{ }^{*} \text { difference }(95 \% \\
\text { Cl), P value }\end{array}$ & Crude mean (SD) & $\begin{array}{l}\text { Adjusted difference }(95 \% \\
\text { CI), P value }\end{array}$ \\
\hline $\begin{array}{l}\text { Whole cohort } \\
(n=743)\end{array}$ & $1.67(0.82)$ & $1.71(0.96)$ & $0.04(-0.11$ to 0.19$), 0.59$ & $1.78(0.94)$ & 0.11 (-0.04 to 0.26$), 0.14$ \\
\hline LRTI $(n=113)$ & $2.14(0.99)$ & $1.70(0.94)$ & $-0.40(-0.78$ to -0.01$), 0.04$ & $1.74(0.84)$ & $-0.47(-0.84$ to -0.10$), 0.01$ \\
\hline Non-LRTI $(n=630)$ & $1.60(0.77)$ & $1.73(0.97)$ & 0.11 ( -0.05 to 0.27$), 0.19$ & $1.77(0.96)$ & 0.20 (0.03 to 0.36$), 0.03$ \\
\hline Age $\leq 16(n=200)$ & $1.74(0.80)$ & $1.20(0.79)$ & $-0.47(-0.76$ to -0.18$),<0.01$ & $1.61(0.90)$ & $-0.04(-0.31$ to 0.23$), 0.77$ \\
\hline Age $\geq 17(n=543)$ & $1.65(0.83)$ & $1.88(0.96)$ & 0.20 (0.03 to 0.38 ), 0.02 & $1.85(0.95)$ & 0.16 (-0.02 to 0.34$), 0.08$ \\
\hline
\end{tabular}

*Adjusted for baseline symptom severity, dosing, steam, antibiotic prescribing, and smoking (as smoking significantly predicted symptom severity). Outcome is severity of symptoms so lower symptom severity (or negative change) is better. Denominators vary because of missing data. 
Table 3| Effectiveness of advice strategies for steam inhalation for symptom control (symptom severity in two to four days after seeing doctor) in patients with acute respiratory tract infections

\begin{tabular}{|c|c|c|c|}
\hline & \multicolumn{2}{|c|}{ Crude mean (SD) } & \multirow[b]{2}{*}{ Adjusted $^{\star}$ difference $(95 \% \mathrm{Cl}), \mathrm{P}$ value } \\
\hline & No steam & Steam & \\
\hline Whole cohort $(n=743)$ & $1.74(0.92)$ & $1.70(0.90)$ & $-0.02(-0.14$ to 0.10$), 0.75$ \\
\hline LRTI $(n=113)$ & $1.93(0.96)$ & $1.75(0.90)$ & $0.03(-0.27$ to 0.33$), 0.86$ \\
\hline Non-LRTI ( $\mathrm{n}=630)$ & $1.71(0.91)$ & $1.68(0.89)$ & $-0.01(-0.14$ to 0.13$), 0.93$ \\
\hline Age $\leq 16(n=200)$ & $1.49(0.79)$ & $1.57(0.92)$ & $0.08(-0.14$ to 0.31$), 0.46$ \\
\hline Age $\geq 17(n=543)$ & $1.82(0.94)$ & $1.75(0.89)$ & $-0.06(-0.20$ to 0.09$), 0.44$ \\
\hline
\end{tabular}

*Adjusted for baseline symptom severity, dosing, steam, antibiotic prescribing, and smoking (as smoking significantly predicted symptom severity). Outcome is severity of symptoms so lower symptom severity (or negative change) is better. Denominators vary because of missing data. 
Table 4| Reported harms associated with interventions for respiratory tract infections. Figures are numbers (percentage) of patients

\begin{tabular}{lccc} 
& Paracetamol & Ibuprofen & Combined \\
Diarrhoea & $29 / 150(19)$ & $20 / 150(13)$ & $16 / 134(12)$ \\
\hline Vomiting & $23 / 150(15)$ & $16 / 149(11)$ & $18 / 135(13)$ \\
\hline Abdominal pain & $39 / 149(26)$ & $39 / 147(27)$ & $42 / 132(32)$ \\
\hline Skin rash & $6 / 150(4)$ & $10 / 149(7)$ & $9 / 134(7)$ \\
\hline Wheeze & $56 / 150(37)$ & $59 / 152(39)$ & $52 / 134(39)$ \\
\hline
\end{tabular}


Table 5| Effectiveness of advice strategies for analgesia on other outcomes in patients with acute respiratory tract infections

\begin{tabular}{|c|c|c|c|c|c|}
\hline & \multirow[b]{2}{*}{$\begin{array}{l}\text { Paracetamol } \\
\text { crude data }\end{array}$} & \multicolumn{2}{|c|}{ Ibuprofen } & \multicolumn{2}{|c|}{ Paracetamol and ibuprofen } \\
\hline & & Crude data & $\begin{array}{c}\text { Adjusted }{ }^{*} \text { statistic }(95 \% \\
\text { CI), P value }\end{array}$ & Crude mean (SD) & $\begin{array}{l}\text { Adjusted* statistic }(95 \% \\
\text { CI), P value }\end{array}$ \\
\hline $\begin{array}{l}\text { Crude mean (SD) temperature } \\
\text { days } 2-4(n=439)\end{array}$ & $36.6(0.6)$ & $36.6(0.6)$ & $\begin{array}{c}0.07 \dagger(-0.06 \text { to } 0.19) \\
0.301\end{array}$ & $36.6(0.5)$ & $\begin{array}{c}-0.07 \dagger(-0.20 \text { to } 0.06) \\
0.271\end{array}$ \\
\hline $\begin{array}{l}\text { Crude median }(25-7 \text { th centile) } \\
\text { duration of symptoms rated } \\
\text { moderately bad or worse } \\
(n=722)\end{array}$ & 4 (3 to 7 ) & 4 (3 to 7$)$ & $0.97 \ddagger(0.79$ to 1.19$), 0.768$ & 4 (3 to 7$)$ & $0.98 \ddagger(0.80$ to 1.20$), 0.853$ \\
\hline No (\%) using antibiotic $(n=736)$ & $145 / 251(58)$ & $145 / 254(57)$ & $0.99 \S(0.79$ to 1.17$), 0.891$ & $140 / 239(59)$ & $1.02 \S(0.83$ to 1.21$), 0.803$ \\
\hline $\begin{array}{l}\text { No }(\%) \text { reconsultation (with new } \\
\text { or unresolved symptoms or } \\
\text { complications within } 1 \text { month) }\end{array}$ & $35 / 300(12)$ & $58 / 295(20)$ & $1.67 \S$ ( 1.12 to 2.38$), 0.012$ & $48 / 285(17)$ & $1.49 \S(0.98$ to 2.18$), 0.058$ \\
\hline $\begin{array}{l}\text { No }(\%) \text { who believe in } \\
\text { effectiveness of antibiotics }\end{array}$ & $116 / 138(84)$ & $112 / 138(81)$ & $0.95 \S(0.80$ to 1.05$), 0.384$ & $100 / 129(78)$ & $0.90 \S(0.74$ to 1.02$), 0.123$ \\
\hline No (\%) very satisfied with & $82 / 96(85)$ & $83 / 97(86)$ & $1.00 \S(0.84$ to 1.09$), 0.986$ & $77 / 88(88)$ & $1.02 \S(0.86$ to 1.10$), 0.791$ \\
\hline
\end{tabular}

*Models all controlled for baseline symptom severity, dosing, steam, antibiotic prescribing and smoking; duration model also controlled for previous duration of illness; temperature model also controlled for baseline temperature; model for return with unresolved symptoms also controlled for initial diagnosis. Denominators vary because of missing data.

†Adjusted difference.

$\ddagger$ Hazard ratio.

§Risk ratio. 
Table 6 | Effectiveness of advice strategies for steam inhalation on other outcomes in patients with acute respiratory tract infections

\begin{tabular}{|c|c|c|c|}
\hline & No steam & Steam & Adjusted $^{*}$ statistic $(95 \% \mathrm{Cl}), \mathrm{P}$ value \\
\hline Crude mean (SD) temperature days $2-4(n=439)$ & $36.6(0.6)$ & $36.6(0.6)$ & $0.05 \dagger(-0.05$ to 0.16$), 0.32$ \\
\hline $\begin{array}{l}\text { Crude median ( } 25-7 \text { th centile) duration of symptoms } \\
\text { rated moderately bad or worse }(n=722)\end{array}$ & 4 (3 to 7$)$ & 4 (3 to 7 ) & $1.04 \ddagger$ (0.89 to 1.23$), 0.61$ \\
\hline No (\%) using antibiotic $(n=736)$ & 232/393 (59) & $198 / 351(56)$ & $0.90 \S(0.74$ to 1.06$), 0.211$ \\
\hline $\begin{array}{l}\text { No (\%) reconsultation (with new or unresolved } \\
\text { symptoms or complications within } 1 \text { month) }\end{array}$ & $69 / 453(15)$ & $72 / 427(17)$ & $1.10 \S(0.79$ to 1.51$), 0.55$ \\
\hline No (\%) who believe in effectiveness of antibiotics & $179 / 220(81)$ & $149 / 185(81)$ & $0.98 \S$ (0.87 to 1.05$), 0.61$ \\
\hline No (\%) very satisfied with consultation & 135/154 (88) & $107 / 127(84)$ & $0.95 \S$ (0.79 to 1.05$), 0.41$ \\
\hline
\end{tabular}

*Models all controlled for baseline symptom severity, dosing, steam, antibiotic prescribing, and smoking; duration model also controlled for previous duration of illness; temperature model also controlled for baseline temperature; model for return with unresolved symptoms also controlled for initial diagnosis. Denominators vary because of missing data.

†Adjusted difference.

$\ddagger$ Hazard ratio.

§Risk ratio. 


\section{Figure}

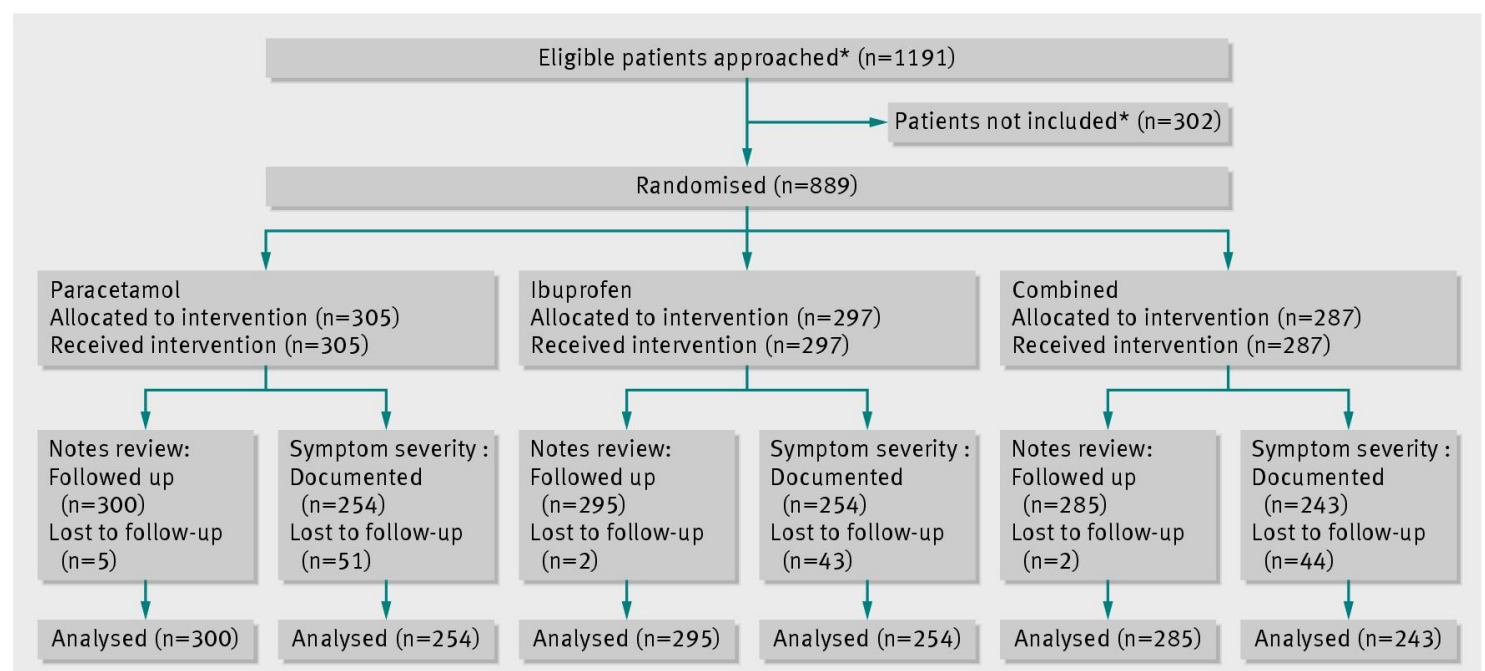

Flow and randomisation of participants. *Figures based on general practitioners' reports 